\title{
Características del empleo en las manufacturas: un análisis a nivel nacional y para el Estado de México, 2005-2019
}

\section{Legbia Pérez Santillán*}

\section{RESUMEN}

El trabajo analiza la relación entre las personas ocupadas en el sector manufacturero y las características específicas de la población (edad, sexo, nivel educativo, entre otras) a nivel nacional y en el Estado de México entre el primer trimestre de 2005 y el tercero de 2019, con base en datos de la Encuesta Nacional de Ocupación y Empleo (ENOE) del INEGI. Se plantea una estimación basada en un pseudopanel y un estimador de efectos fijos desarrollados mediante la construcción de las cohortes asociados al año de nacimiento. Los resultados muestran variables como el empleo formal, el nivel de ingreso, el tamaño de los establecimientos y la edad de las personas como aspectos que se asocian positiva o negativamente con las personas ocupadas en el empleo manufacturero.

Palabras clave: empleo manufacturero, características del empleo, Estado de México, pseudopanel.

Clasificación JEL: J21, J24, R11, C23.

* Profesora investigadora de la Universidad Autónoma del Estado de México, México. Correo electrónico: lperezs@uaemex.mx. 


\section{ABSTRACT \\ Characteristics of Employment in Manufacturing: an Analysis at the National Level and for the Estado de México, 2005-2019}

This paper analyzes the relationship between people employed in the manufacturing sector and specific characteristics of the population (age, sex, educational level, among others) at the national level and in the Estado de Mexico between the first quarter of 2005 and the third of 2019. The INEGI's National Occupation and Employment Survey (ENOE) is used as a source of information. The study proposes an estimate based on a pseudo panel and a fixed effects estimator developed by constructing the cohorts associated with the year of birth. The results show variables such as formal employment, income level, size of establishments, and people's age as aspects that are positively or negatively associated with people employed in manufacturing employment.

Keywords: Manufacturing employment, employment characteristics, State of Mexico, Pseudo panel.

JEL Classification: J21, J24, R11, C23.

\section{INTRODUCCIÓN}

Una de las variables clave en el seguimiento del desempeño económico es el empleo. El crecimiento de la fuerza laboral, como factor de la producción, contribuye al económico; en tanto, la economía requiere insertarse en una senda de crecimiento sostenible para generar suficientes puestos de trabajo y absorber o incorporar a las personas que ingresan al mercado laboral.

En los momentos de expansión o auge económico, la preocupación suele centrarse en la cantidad de empleos que se generan y en los de contracción o recesión económica, en aquellos puestos de trabajo que se pierden. Los estudios empíricos llevaron a establecer la conocida Ley de Okun que retomaron de la relación entre las variaciones en la producción y el desempleo en Estados Unidos encontrada por Okun (1962). Además de la producción, en los mercados laborales, se reconoce que existen numerosos determinantes del nivel de empleo (o de desempleo), entre los cuales sobresalen algunas características de la persona, como: edad, experiencia laboral, años de escolaridad, sexo, ámbito de residencia (rural o urbano) entre otras (Varela y Nava, 2015; 
Llamas y Garro, 2003). Al estudio de la diferencia entre el ritmo al que crece una economía y al que cambia el nivel de empleo, se añaden entonces los análisis relacionados con los cambios en los tipos de empleos, formales, informales, con acceso a la seguridad social, entre otros y sus posibles determinantes (Martínez Luis et al., 2018).

Al respecto, Vázquez (2018) menciona que, dentro de las actuales prioridades de las agendas nacionales e internacionales, tanto en los países en desarrollo como en los desarrollados, está alcanzar crecimiento inclusivo y la creación de empleo productivo. Mientras que para los hacedores de política, así como para las organizaciones de trabajadores y empleadores, el Objetivo de Desarrollo Sostenible (ODS) núm. 8 de la Agenda de Desarrollo Sostenible 2030, plantea tener un mejor entendimiento del funcionamiento del mercado laboral. El mencionado ODS promueve los esfuerzos para impulsar el crecimiento económico sostenible, inclusivo y sostenido, empleo pleno y productivo, y trabajo decente para todos. Para lograr esos objetivos en el mercado laboral se requiere el manejo de las fuentes estadísticas relacionadas con el empleo y sus indicadores, como elementos previos para el diseño de políticas públicas de fomento al empleo.

Por consiguiente, este documento tiene como objetivo mostrar cómo se relacionan a lo largo del tiempo las características de la población en general y de personas ocupadas en el sector manufacturero en México y en el Estado de México. Aunque las actividades manufactureras han dejado su lugar protagónico con base en su participación en la producción y empleos totales, el sector es relevante por sus vínculos con otros sectores y por su presencia en entidades como el Estado de México, por ello es pertinente el examen de temas relacionados con el empleo generado por estas actividades.

Para lograr el objetivo, el documento se organiza de la siguiente manera. Posterior a la introducción se presentan cuatro apartados. En el primero se describe y analiza la evolución del empleo total, manufacturero nacional y del Estado de México de 2005 a 2019. En el segundo se expone la metodología y estimaciones; en el tercero se discuten los resultados. Finalmente se presentan las conclusiones. 


\section{EVOLUCIÓN DEL EMPLEO TOTAL Y MANUFACTURERO, NACIONAL Y EN EL ESTADO DE MÉXICO, 2005-2019}

Entre el primer trimestre de 2005 y de 2019, de acuerdo con datos de la Encuesta Nacional de Ocupación y Empleo (ENOE) del INEGI, en México la población total pasó de 106.7 a 125.5 millones de personas, un incremento de 18.8 millones de personas (o de 17.6\%) en el periodo. ${ }^{1}$ En cuanto a la población de 15 años o más, pasó de 73.1 a 94.3 millones, un aumento de 21.2 millones de personas (o de $29.0 \%$ ) en el mismo periodo. Por su parte, la población económicamente activa (PEA) se incrementó en 12.9 millones de personas (30\%), al pasar de 43.1 a 56.0 millones. Esto equivale a aproximadamente 924 mil personas que cada año se incorporaron a la PEA de 2005 a 2019.

Por lo que se refiere al Estado de México, la población pasó de 14.1 a 17.8 millones de personas, un incremento de 3.6 millones de personas (o 25.6\%) en el periodo. En este lapso, la entidad se caracteriza por tener la mayor participación en la población total $(14.1 \%$ en el primer trimestre de 2019), además de un crecimiento superior al nacional. Por su parte, la población de 15 años o más pasó de 9.8 a 13.6 millones; esto es, 3.8 millones de personas más (39.1\%) en el mismo periodo.

La población económicamente activa (PEA) creció en 2.1 millones de personas, al pasar de 5.7 a 7.9 millones. Para la entidad significó que aproximadamente cada año 150 mil personas se incorporaron a la PEA de 2005 a 2019.

En el ámbito nacional, la población ocupada (PO) en el periodo señalado pasó de 41.4 a 54.2 millones de personas ${ }^{2}$ (un incremento de $30.7 \%$ o 12.7 millones de personas). De esta manera, el incremento en la PEA en ese lapso fue ligeramente superior al de la población ocupada (908 mil en promedio por año) y el número de personas desocupadas ha aumentado en aproximadamente 200 mil personas y se ubica en alrededor de 1.9 millones de personas en el primer trimestre de 2019. En el

Ocho entidades concentraban poco más de la mitad de la población total del país en 2005 y mantuvieron la participación en 2019: Estado de México, Ciudad de México, Veracruz, Jalisco, Puebla, Guanajuato, Chiapas y Nuevo León.

2 Sobresale que entre el cuarto trimestre de 2012 y el mismo trimestre de 2017 la población ocupada se incrementó en alrededor de 4 millones de personas. 
Estado de México, la PO se incrementó de 5.5 a 7.6 millones de personas (2.1 millones de personas o 37.5\%). Durante los primeros trimestres tanto de 2005 como de 2019, al igual que en el ámbito nacional, la población desocupada prácticamente no cambió y se ubicó en alrededor de 339 mil personas.

Entre el primer trimestre de 2005 y el tercer trimestre del 2019, el total de personas ocupadas por sector de actividad económica se distribuyó como se muestra en la Gráfica 1. El sector primario, que incluye las actividades de agricultura, ganadería, silvicultura, caza y pesca concentró en promedio $13.5 \%$ del total de personal ocupado; el sector secundario integrado por las actividades de la industria extractiva y de la electricidad, industria manufacturera y construcción aportó en promedio $24.8 \%$ del empleo total, mientras que el sector terciario, conformado por actividades de comercio; restaurantes y servicios de alojamiento; transportes, comunicaciones, correo y almacenamiento; servicios profesionales, financieros y corporativos; servicios sociales; servicios diversos; y gobierno y organismos internacionales, concentró en promedio $61.0 \%$ del personal ocupado total. En términos de personas, para el tercer trimestre de $2019,{ }^{3}$ del total de personas ocupadas (54.9 millones), 7.0 millones se ocupaban en el sector primario, 13.9 millones en el secundario y 33.9 millones en el terciario. Por lo tanto, la población ocupada en el sector manufacturero ascendió a 9.1 millones.

3 En el primer trimestre de 2005, del total de personas ocupadas (41.4 millones), 6.1 millones de personas se ocupaban en el sector primario, 10.7 millones en el secundario, y 24.3 millones en el terciario. El total de personas ocupadas en el sector manufacturero ascendió a 7.1 millones de personas. 
GRÁFICA 1

MÉXICO, DISTRIBUCIÓN DE LAS PERSONAS OCUPADAS POR SECTOR DE ACTIVIDAD ECONÓMICA 2005.I- 2019.III (PORCENTAJE)

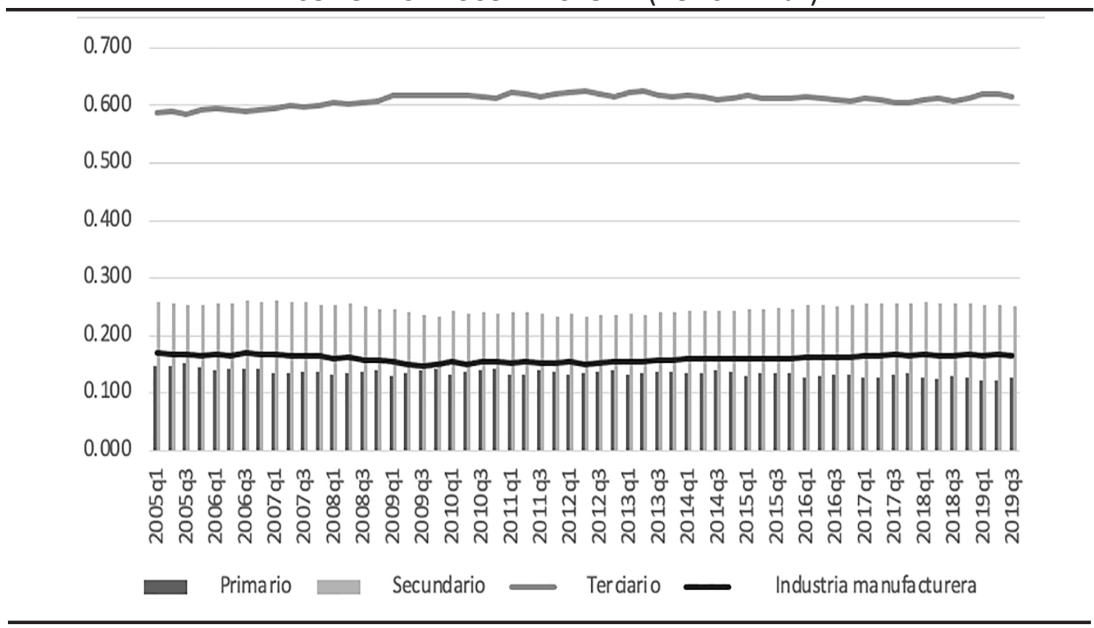

Nota: no se incluyen a las personas con sector de actividad no especificado.

Fuente: elaborado con datos de la ENOE.

Entre el primer trimestre de 2005 y el mismo trimestre de 2019, las personas ocupadas en el sector manufacturero pasaron de 7.1 a 8.9 millones; hubo un incremento de 26.9\% (1.9 millones de personas). En términos relativos, el crecimiento del empleo manufacturero fue menor al observado para el total del empleo (30.7\%), para el sector terciario $(38.2 \%)$ y para el total del sector secundario $(27.5 \%)$, y sólo estuvo por arriba del crecimiento en el sector primario $(8.4 \%)$. Por otra parte, el crecimiento del empleo manufacturero representó sólo $15.0 \%$ del incremento total en el empleo, de manera que su participación pasó de aportar $17.1 \%$ a $16.6 \%$ del empleo total.

En el Estado de México, durante el periodo de estudio, las personas ocupadas en manufacturas pasaron de 1.13 a 1.36 millones, equivalente a un incremento de $21.0 \%$ (237 mil personas), porcentaje menor al incremento nacional. En términos relativos, el crecimiento del empleo manufacturero fue menor al observado para el total de éste (37.5\%) $\mathrm{y}$, ese crecimiento representó sólo $11.5 \%$ del incremento total en el empleo, por lo que la participación del empleo manufacturero en la entidad pasó de $20.5 \%$ a $18.1 \%$ del empleo total; pese al descenso, aún está por encima de la participación a nivel nacional. 
GRÁFICA 2

ESTAdO dE MÉxICO, DISTRIBUCIÓN DE LAS PERSONAS OCUPADAS POR SECTOR DE ACTIVIDAD ECONÓMICA 2005.I-2019.III (PORCENTAJE)

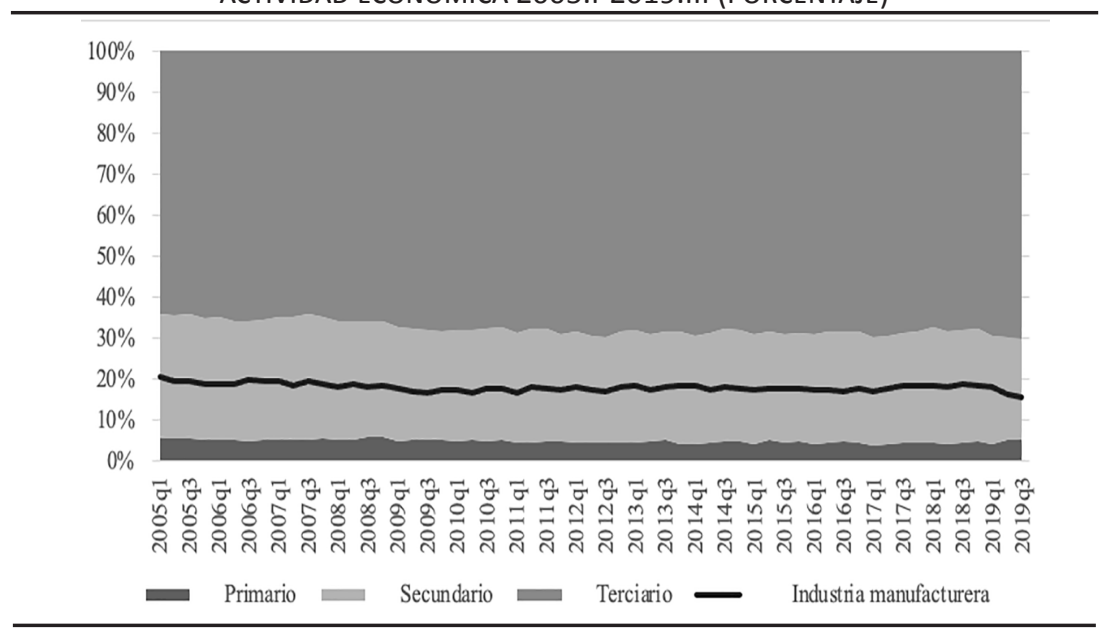

Nota: no se incluyen las personas con sector de actividad no especificado.

Fuente: elaborado con datos de la ENOE.

Como se muestra en la Gráfica 2, la estructura del empleo en el Estado de México es, en general, similar a la nacional, aunque se distinguen mayores participaciones de los sectores secundario (y de las manufacturas) y terciario, así como una menor participación del sector primario en el empleo.

En el primer trimestre de 2005, el empleo manufacturero se concentró (alrededor de 3.9 millones de personas ocupadas o 54.5\%) en el Estado de México (16.0\%); Jalisco (7.9\%); Ciudad de México (7.9\%); Guanajuato (6.3\%); Nuevo León (6.0\%); Puebla (5.9\%) y Chihuahua (4.6\%). Para el mismo trimestre de 2019, las mismas entidades concentraban el empleo manufacturero (4.7 millones de empleos o 52.6\%): Estado de México (15.2\%); Jalisco (7.7\%); Guanajuato (7.9\%); Nuevo León (6.4\%); Chihuahua (5.3\%); Puebla (5.5\%) y Ciudad de México (4.5\%).

Al igual que con el empleo total, el manufacturero presenta un comportamiento distinto entre entidades federativas: seis concentraron $58.5 \%$ del incremento en las personas ocupadas del sector (alrededor de 1.1 millones de personas): Estado de México (12.5\%); Guanajuato (14.0\%); Chihuahua (8.1\%); Nuevo León (7.9\%); Coahuila (8.5\%) y Baja California (7.5\%). Se distingue que, en el periodo referido, cuatro entidades perdieron alrededor de 177 mil personas ocupadas en el 
sector manufacturero: Veracruz (-1.1 mil empleos); Sinaloa (-2.4 mil); Michoacán (-25 mil) y la Ciudad de México (-150 mil).

La evolución de los niveles de empleo y desempleo ha estado ligada al desempeño económico. De acuerdo con Carbajal y de Jesús (2017), los episodios de crisis en la economía mexicana han hecho evidente la dificultad posterior de recuperar los niveles de actividad económica y de empleo. Así, debe tenerse en cuenta que el número de personas desocupadas se incrementó a partir del tercer trimestre de 2008 (al alcanzar aproximadamente 2 millones de personas) y alcanzó su máximo en el tercer trimestre de 2009 (casi 3 millones de personas desocupadas). A partir del cuarto trimestre de 2009, el número de personas desocupadas comenzó a descender en la mayoría de los trimestres siguientes. En recientes trimestres se han alcanzado cifras similares a las de 2008 (alrededor de 1.8 millones de personas desocupadas en promedio).

En términos de la tasa de desocupación, en los últimos cuatro trimestres ha sido en promedio de $3.5 \%$, nivel similar al observado en promedio en los trimestres de 2007 y los primeros dos de 2008. Del primer trimestre de 2005 al mismo periodo, pero de 2019, el nivel máximo de la tasa de desocupación fue de $6.15 \%$ en el tercer trimestre de 2009, y a partir de los siguientes trimestres prevalecieron los descensos hasta alcanzar niveles inferiores a $4 \%$ a partir del tercer trimestre de 2016.

La dinámica de la población ocupada y la generación de empleos son distintas entre entidades federativas. Entre el primer trimestre de 2005 y el mismo periodo, pero de 2019, el Estado de México se encontró dentro del grupo de entidades en las que el crecimiento del PIB y de la población ocupada superaron a lo observado para esos indicadores en el ámbito nacional (Pérez Santillán, 2019a). En específico, en el Estado de México en promedio la tasa de crecimiento del PIB en el periodo fue de $2.89 \%$ (2.28 en el ámbito nacional) y la tasa de crecimiento de la población ocupada de $2.43 \%$ ( $1.84 \%$ nacional). Por lo que se refiere al sector manufacturero, en el Estado de México en promedio la tasa de crecimiento del PIB en el periodo fue de $0.80 \%$ (1.81 en el ámbito nacional) y la tasa de crecimiento de la población ocupada de $2.34 \%(1.98 \%$ nacional). Si bien a nivel nacional se observa relación entre el crecimiento económico y la generación de empleo (Gráfica 3), ésta mejora al concentrar el análisis en la producción y empleo manufactureros. 
GRÁFICA 3

MÉXICO: TASA DE CRECIMIENTO DEL PIB Y EMPLEO MANUFACTURERO 2005-2018 (PORCENTAJE)

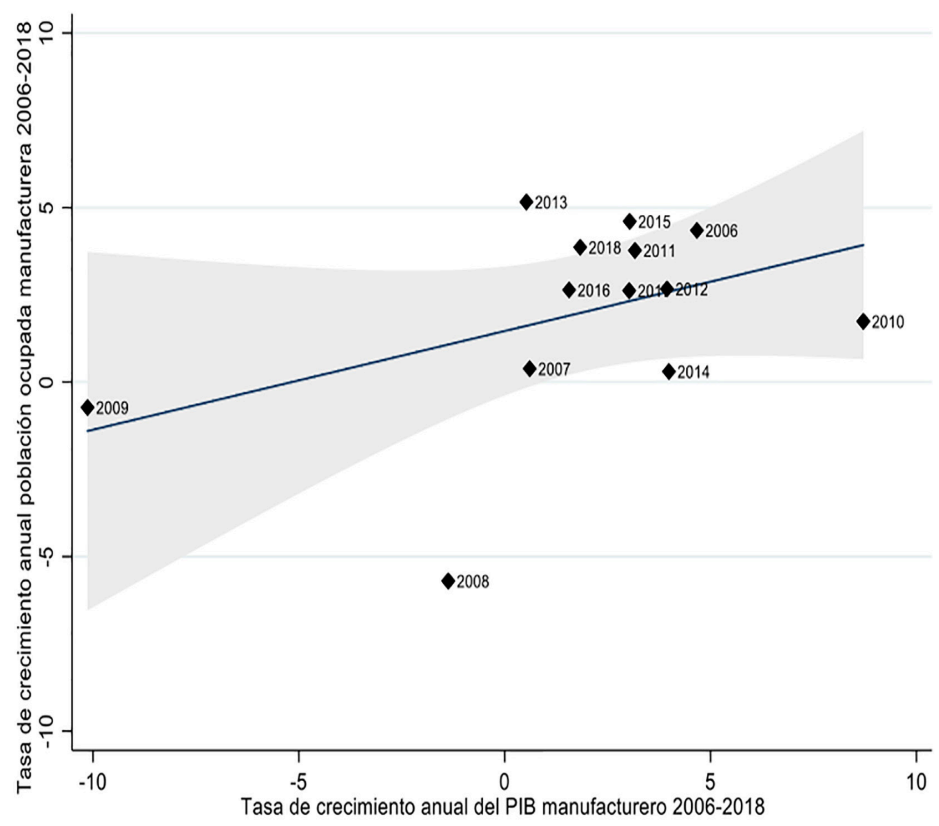

Fuente: elaborado con datos del INEGI.

Cabe señalar que el comportamiento de la población ocupada en las manufacturas y el crecimiento del PIB manufacturero exhiben diferencias entre el agregado nacional y el correspondiente al Estado de México. La producción y empleo manufactureros registraron decrementos como resultado de la gran recesión de 2008-2009, pero en el ámbito nacional estos indicadores reportaron tasas positivas luego de esa coyuntura. En cambio, en el Estado de México el menor crecimiento del PIB manufacturero se debe a que incluso después de la mencionada crisis, varios años presentó tasas negativas tanto en la producción como en el empleo (por ejemplo, en 2014 y 2016). De ahí que la dispersión en el crecimiento del PIB manufacturero sea mayor en el Estado de México en relación con lo observado en el ámbito nacional (Gráfica 4). 
GRÁFICA 4

ESTAdO de MÉXICO: TASA DE CRECIMIENTO DEL PIB Y EMPLEO MANUFACTURERO 20052018 (PORCENTAJE)

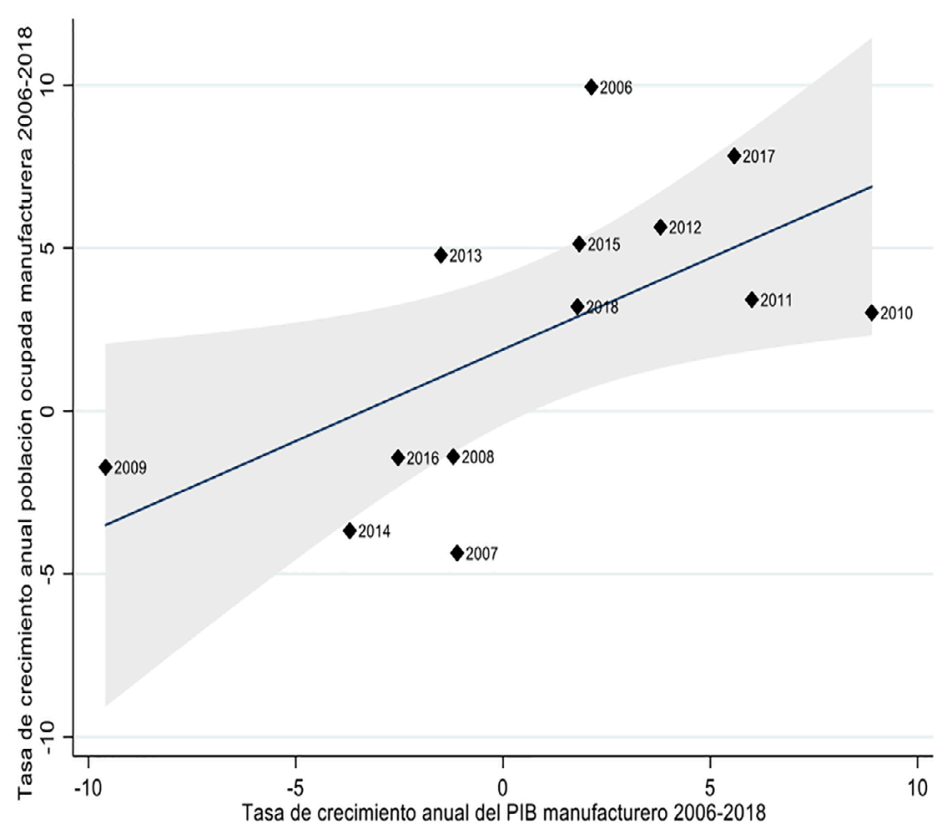

Fuente: elaborado con datos del INEGI.

En la relación positiva entre el crecimiento de la producción manufacturera y el empleo en el sector se espera que el incremento de la producción manufacturera genere un aumento en la cantidad demandada de fuerza laboral. Lo anterior corresponde con lo que McConell y Macpherson (2003) denominan a la demanda de fuerza laboral como una demanda derivada o determinada por el nivel de producción.

Además del nivel de producción, existen diversos determinantes de la oferta y demanda de fuerza laboral. Una revisión reciente de las características del empleo y la producción manufacturera, desagregada por sectores de actividad y entidad federativa, se encuentra en Carbajal y Carrillo (2017). De igual manera, en Dussel Peters y Pérez Santillán (2018) se revisan las principales características del empleo manufacturero en México. En ambos documentos, se describen peculiaridades del total de personas ocupadas en este sector en cuanto a su distribución en actividades y por entidad federativa y en el segundo, se presentan 
aproximaciones a la calidad de los empleos manufactureros. Por su parte, Ríos y Carrillo (2014) analizan el efecto de la crisis de 2009 en el empleo manufacturero mediante la desagregación por ramas o subsectores; además, examinan los cambios en ese nivel del empleo calificado y no calificado asociado al nivel tecnológico de las ramas o subsectores, no a partir de las características de las personas.

Cabe destacar que Carbajal y Carrillo (2017) afirman que existen numerosos trabajos sobre el sector manufacturero a nivel agregado, y otro menor que se encuentran por entidades federativas y en menor cuantía si se les relaciona con la generación de empleos. En este documento, la atención se centra en las características de las personas en edad de trabajar y su relación con la población ocupada manufacturera. De acuerdo con la revisión realizada, son escasos los trabajos empíricos que analicen la incidencia de las características de las personas (en cuanto a edad, escolaridad, ámbito de residencia, entre otras) en su decisión de ocuparse en las actividades manufactureras. Una descripción breve muestra que a nivel nacional el total de población ocupada en manufacturas pasó de 7.08 a 8.98 millones de personas entre el primer trimestre de 2005 y el mismo trimestre, pero de 2019 (Cuadro 1). En el primer trimestre de 2005, 38.02\% (37.1\% en 2019.1) eran mujeres y $61.98 \%$ (62.9\%) hombres. Para el primer trimestre de 2005 , $75.8 \%(74.7 \%)$ tenían de 20 a 49 años; $10 \%$ (5.9\%) de 14 a 19 años y $14.2 \%$ (19.4\%) 50 o más años.

Por lo que se refiere a la escolaridad, en el primer semestre de 2005, $20.37 \%$ (13.25\%) de las personas ocupadas en las manufacturas tenían 6 años de escolaridad (primaria); $28.60 \%$ (34.2\%) 9 años (secundaria) y $12.61 \%$ (20.38\%) 12 años (preparatoria o bachillerato). En el periodo considerado, la escolaridad promedio pasó de 8.4 a 9.9 años.

En cuanto a la formalidad laboral, ${ }^{4}$ en el primer trimestre de 2005, $42.63 \%(37.61 \%)$ de las personas ocupadas en las manufacturas eran informales y $57.37 \%(62.39 \%)$ formales. Por lo que se refiere al estado

4 Se refiere a los ocupados en el sector formal que el INEGI define como: "todas aquellas actividades económicas que se desarrollan en empresas constituidas en sociedad, corporaciones, instituciones públicas o privadas, negocios no constituidos en sociedad, pero que reportan ingresos ante la autoridad hacendaria y en la agricultura y/o crianza de animales orientadas al mercado". 
conyugal, 48.93\% (43.58\%) eran casados; $33.2 \%$ (29.8\%) solteras y solteros, y $10.44 \%$ (18.09\%) estaban en unión libre. Además, $11.2 \%$ (7.4\%) estaban en pequeños establecimientos, $18.19 \%(15.56 \%)$ en medianos y $24.33 \%$ (33.11\%) en grandes.

\section{CUADRO 1}

CARACTERÍSTICAS SELECCIONADAS DE LA POBLACIÓN OCUPADA MANUFACTURERA, 2005.I Y 2019.1

\begin{tabular}{|c|c|c|}
\hline & 2005.1 & 2019.1 \\
\hline Total (millones de personas) & 7.08 & 8.98 \\
\hline \multicolumn{3}{|l|}{ Sexo (porcentaje) } \\
\hline Mujer & 38.02 & 37.13 \\
\hline Hombre & 61.98 & 62.87 \\
\hline \multicolumn{3}{|l|}{ Edad (años) } \\
\hline De 14 a 19 años & 10.04 & 5.89 \\
\hline De 20 a 29 años & 31.06 & 26.30 \\
\hline De 30 a 39 años & 26.80 & 25.27 \\
\hline De 40 a 49 años & 17.92 & 23.09 \\
\hline De 50 a 59 años & 9.11 & 13.71 \\
\hline De 60 años y más & 5.07 & 5.68 \\
\hline Edad no especificada & 0.01 & 0.06 \\
\hline \multicolumn{3}{|l|}{ Estado conyugal (porcentaje) } \\
\hline Unión libre & 10.44 & 18.09 \\
\hline Separado(a) & 3.53 & 4.53 \\
\hline Divorciado(a) & 1.35 & 1.68 \\
\hline Viudo(a) & 2.54 & 2.32 \\
\hline Casado(a) & 48.93 & 43.58 \\
\hline Soltero(a) & 33.20 & 29.80 \\
\hline \multicolumn{3}{|c|}{ Tamaño de establecimiento (porcentaje) } \\
\hline No aplica & 1.41 & 4.79 \\
\hline Sin establecimiento & 19.00 & 17.06 \\
\hline Con establecimiento & 25.86 & 22.09 \\
\hline Pequeños establecimientos & 11.20 & 7.40 \\
\hline Medianos establecimientos & 18.19 & 15.56 \\
\hline Grandes establecimientos & 24.33 & 33.11 \\
\hline \multicolumn{3}{|l|}{ Nivel de ingresos (porcentaje) } \\
\hline Hasta un salario mínimo & 12.0 & 17.29 \\
\hline 1 hasta 2 salarios mínimos & 29.4 & 38.02 \\
\hline 2 hasta 3 salarios mínimos & 25.6 & 20.21 \\
\hline 3 hasta 5 salarios mínimos & 16.2 & 7.2 \\
\hline Más de 5 salarios mínimos & 8.5 & 2.7 \\
\hline No recibe ingresos & 4.4 & 2.97 \\
\hline No especificado & 4.0 & 11.62 \\
\hline Años de escolaridad promedio & 8.4 & 9.9 \\
\hline
\end{tabular}

Fuente: elaborado con datos de la ENOE-INEGI de 2005.I y 2019.I. 
En Pérez Santillán (2019b) se presentan resultados basados en modelos logísticos multinomiales para los primeros trimestres de 2005 y 2018; evaluado en la media de la edad, indican que a mayor edad disminuye la probabilidad de ser ocupado manufacturero, mientras que en 2005 ser del sexo masculino incrementó la probabilidad de serlo y en 2018, disminuyó. Adicionalmente, el efecto marginal evaluado en la media de los años de escolaridad indica que un año adicional disminuye la probabilidad de ser ocupado manufacturero, de acuerdo con los modelos para 2005 y 2018, respectivamente.

Por otra parte, en 2005 las personas en situación conyugal soltero (a), separado (a) o viudo (a) presentan una mayor probabilidad de ser ocupado manufacturero comparados con aquellas personas en unión libre. Para 2018, ser soltero o viudo disminuyó la probabilidad de ser ocupado manufacturero. Otro resultado relevante es que para 2005 y 2018 las personas con ingresos mayores a un salario mínimo presentan una menor probabilidad de ser ocupadas manufactureras, comparadas con aquellas con ingresos de hasta un salario mínimo. Finalmente, para 2005 y 2018, a mayor tamaño de establecimiento, se incrementó la probabilidad de ser ocupado manufacturero.

La presente investigación busca ofrecer evidencia empírica de la relación entre un grupo de características de las personas y la ocupación manufacturera mediante la técnica de pseudopanel con un enfoque de cohortes, con objeto de aprovechar la información disponible en la Encuesta Nacional de Ocupación y Empleo (ENOE) del INEGI.

\section{Metodología y estimación}

La fuente de información para este trabajo es la ENOE, encuesta trimestral con datos que van del primer trimestre de 2005 al tercer trimestre de 2019. Como se sabe, la ENOE tiene representatividad nacional y por entidad federativa, y proporciona información sociodemográfica, así como sobre las condiciones de ocupación de las personas. Asimismo, es una encuesta continua trimestral que mantiene un esquema rotatorio en cuanto a sus entrevistados, por lo que se divide en cinco partes; cada parte se visita o entrevista sólo cinco veces, lo que implica que cada trimestre $20 \%$ de la muestra que ya cumplió con su ciclo de cinco visitas, sale de la muestra y se reemplaza. 
Para estudiar la variabilidad de las características de las personas en el mercado laboral lo ideal es apoyarse en bases de datos de tipo longitudinal o panel, así como en aquellas en las que se sigue a individuos o unidades de interés en repetidas ocasiones a través del tiempo. No obstante, su disponibilidad es limitada.

Para superar las limitaciones en cuanto a la información longitudinal, en este trabajo se aprovecha la información de la ENOE para crear pseudopaneles o cohortes sintéticas, como inicialmente lo sugirió Deaton (1985). Los pseudopaneles que se utilizan en la estimación se construyen a partir de la serie temporal de las ENOE trimestrales de 2005 a 2019. El pseudopanel consiste en agrupamientos de las personas con base en criterios que no cambian de una encuesta a otra, en el caso de este trabajo se emplea el año de nacimiento.

La población de interés corresponde a las personas ocupadas en el sector manufacturero. Se consideran 17 cohortes para edades que van de los 18 a los 65 años con años de nacimiento de 1987 a 1939.

El modelo de pseudopanel se define a través de las medias de los subgrupos o cohortes de los $n_{c}$ individuos observados para la cohorte "c" en el periodo " $t$ ". La ecuación básica que representa un modelo de pseudopanel es:

$$
\begin{aligned}
& \bar{y}_{c t}=\overline{\boldsymbol{X}}_{c t} \beta+\bar{a}_{c t}+\bar{\varepsilon}_{c t} \\
& \mathrm{c}=1, \ldots, \mathrm{C} . \quad \mathrm{yt}=1, \ldots, \mathrm{T}
\end{aligned}
$$

Deaton (1985) sugiere que las cohortes construidas a partir de datos de sección transversal repetidos pueden usarse para estimar un modelo de efectos fijos. Las cohortes "c" están definidas por una característica común compartida, de modo que cada individuo es miembro sólo de una cohorte.

En el modelo que se propone aquí, $\overline{\mathrm{y}}_{\mathrm{ct}}$ es el promedio de todas las personas ocupadas en actividades manufactureras en la cohorte $\mathrm{c}$ en el tiempo t y de manera similar para las otras variables en el modelo. Sin embargo, estimar el vector $\beta$ puede ser un problema, porque $\overline{\mathrm{a}}_{\mathrm{ct}}$ depende de $t$ y es probable que esté correlacionado con $\overline{\mathrm{X}}_{\mathrm{ct}}$. Como $\overline{\mathrm{a}}_{\mathrm{ct}}$ no es observable, no se puede incluir directamente en la estimación. Sin embargo, $\overline{\mathrm{a}}_{\mathrm{ct}}$ puede tratarse como un parámetro desconocido fijo a lo largo del tiempo, si existiera un número suficientemente grande 
de observaciones individuales en cada cohorte. Esto hace que la estimación de efectos fijos sea consistente. El vector de parámetros $\beta$ y el efecto fijo por cohorte $\overline{\mathrm{a}}_{\mathrm{ct}}$ pueden ser estimados por mínimos cuadrados ordinarios (MCO). Entonces MCO será consistente mientras el número de personas por cohorte sea grande, como en este caso. En este ejercicio se incluye una estimación con efectos fijos y una estimación pooled para evaluar lo anterior.

Resulta oportuno señalar algunas de las ventajas o bondades de las estimaciones con base en pseudopaneles. Primeramente, suplen la falta de datos con estructura de panel o longitudinales para muchos temas de interés. Además, de acuerdo con Perera et al. (2010), permiten examinar fenómenos dinámicos; en este caso interesa indagar la relación entre cambios en ciertas características de las personas y el empleo manufacturero que no puede ser abordado con información de corte transversal. Asimismo, de acuerdo con los autores y con McKenzie (2004), la metodología de pseudopaneles aminora el sesgo asociado a los errores de medida y distingue cambios asociados a distintas fuentes, como aquellos relacionados con el ciclo de vida, las diferencias intergeneracionales y los ciclos económicos, efectos conocidos como efecto edad, cohorte y temporal, respectivamente.

Las variables explicativas empleadas en los modelos siguientes son: i) Sexo: ii) Años de escolaridad; iii) Ocupados formales en el sector manufacturero; variables relacionadas con la situación conyugal; iv) Unión libre; v) Casado; vi) Soltero y variables relacionadas con el nivel de ingresos en múltiplos de salarios mínimos; vii) Hasta un SM; viii) Más de 1 y hasta 2 SM; ix) Más de 3 y hasta 5 SM; x) Más de 2 y hasta $3 \mathrm{SM}$; xi) Más de 5SM y variables relacionadas con el tamaño del establecimiento y localidad; xii) Pequeños establecimientos (unidades económicas que tienen de 16 a 50 trabajadores en la industria) y xiii) Urbano, que se refiere a localidades con más de 2,500 habitantes. 


\section{RESUltados}

Los datos indican que el promedio de la población ocupada en manufacturas por cohorte tiende a disminuir conforme se incrementa la edad (Gráfica 5). Los porcentajes más elevados de personas por cohorte que están ocupadas en actividades manufactureras se registran en las cohortes 1982/1984; 1979/1981; 1976/1978 y 1973/1975. Por otra parte, el Cuadro 2 muestra estadísticos (promedio) de las variables que se emplearan en los modelos.

\section{GRÁFICA 5}

PERSONAS OCUPAdAS EN MANUFACTURAS EN PROMEDIO POR COHORTE 2005.I A 2019.III

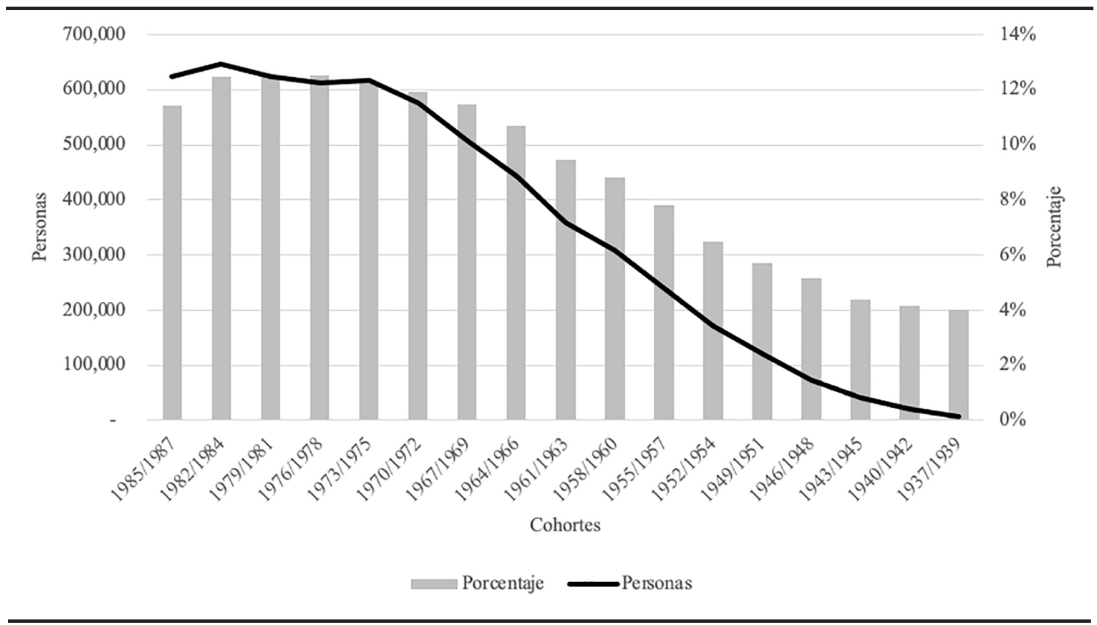

Fuente: elaborado con datos de la ENOE 2005.I a 2019.III 


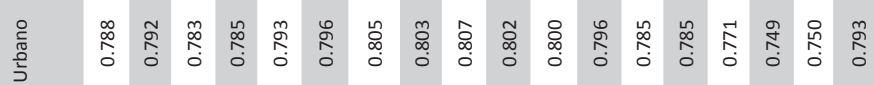

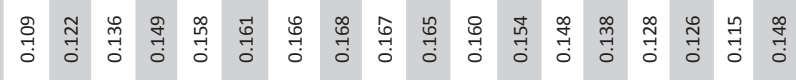$$
\text { ư }
$$$$
\text { 窟 }
$$$$
\text { 竞 }
$$

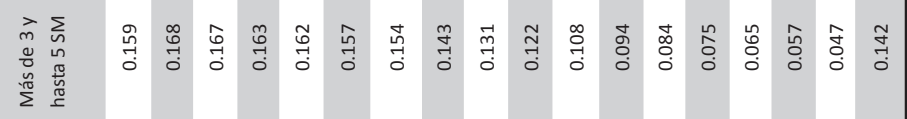

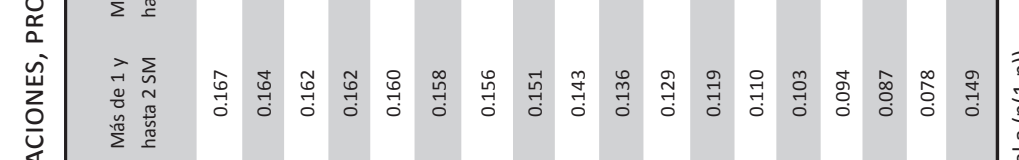

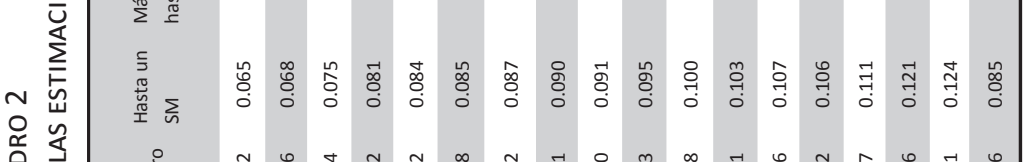

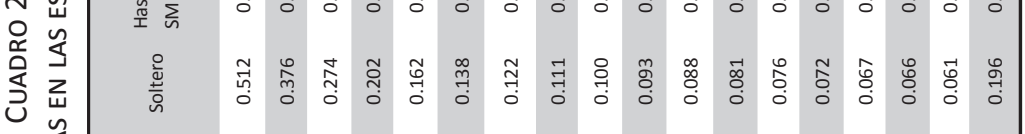

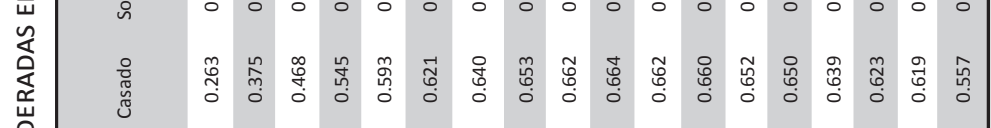

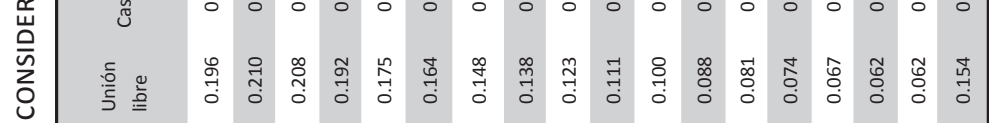

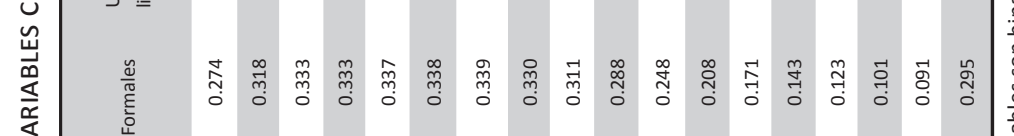

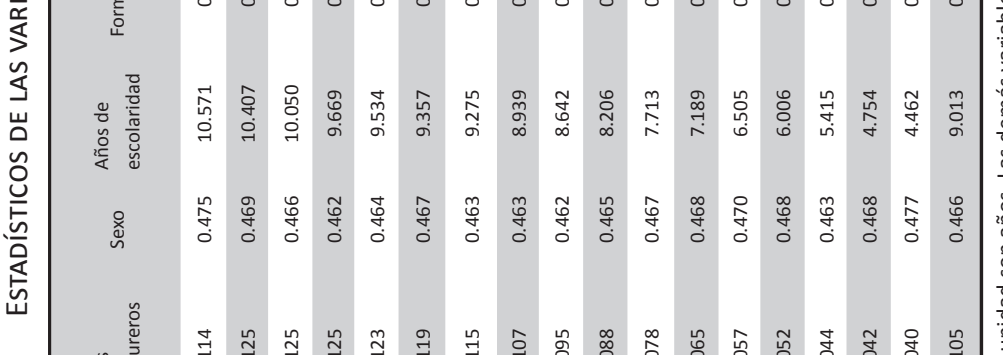

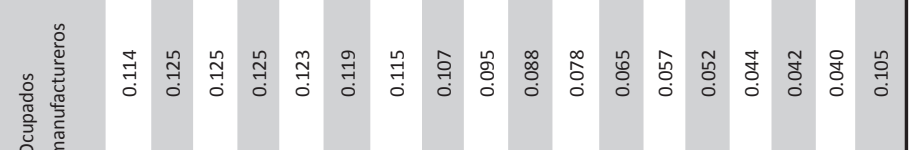
高 
Es importante notar que las características de cada cohorte corresponden al total de población en cada una de ellas. Para las estimaciones se emplean las variables en logaritmos.

A través del pseudopanel se puede seguir el comportamiento promedio de las variables de interés de las cohortes a través del tiempo, en este caso la población ocupada manufacturera. Los promedios se calcularon utilizando datos expandidos. Enseguida se presentan los resultados de la estimación de la relación entre las características promedio de las personas por cohorte y el número de personas ocupadas en manufacturas. Se realizaron regresiones para el total nacional y para el Estado de México.

El Cuadro 3 muestra el resultado de las regresiones. Las tres primeras columnas corresponden a regresiones para el ámbito nacional. La primera columna presenta la estimación de la regresión de la población ocupada manufacturera como datos agrupados, esto es, sin tomar en cuenta la heterogeneidad entre cohortes ni la posible endogeneidad. En la segunda columna se incluyen efectos fijos (asociados a las cohortes) y en la tercera columna se agregan, además de los efectos anteriores, efectos temporales. Las siguientes tres columnas siguen la descripción señalada, con la característica adicional de ser regresiones sólo para la población ocupada manufacturera en el Estado de México.

Dado que se emplean las variables en logaritmos, los resultados de las regresiones se interpretan como elasticidades. En el grupo de regresiones para el ámbito nacional, la regresión agrupada (pool) registra que las variables sexo (indica que un incremento en la proporción o porcentaje de la población de sexo masculino), edad, años de escolaridad, estado conyugal soltero, niveles de ingreso de dos a tres salarios mínimos y de más de cinco salarios mínimos y laborar en una unidad económica con establecimiento tienen una elasticidad negativa y significativa respecto a la población ocupada manufacturera. De otra forma, las características señaladas de la población tienen una relación negativa con la ocupación en las manufacturas. Por otro lado, el empleo formal, tener ingresos de dos y hasta tres salarios mínimos y laborar en unidades económicas que son pequeños establecimientos tienen una elasticidad positiva con respecto a la población ocupada manufacturera.

Una vez que se consideran efectos fijos asociados a las cohortes, se intentan capturar los rasgos de cada cohorte de los sucesivos cortes transversales y se supone que las diferencias en la población ocupada 
manufacturera en cada cohorte no son aleatorias, sino constantes o fijas. Para este modelo, a partir de la ecuación 1, se permite que cada intercepto sea distinto, por lo que es necesario agregar un vector de variables dicotómicas para cada cohorte.

Para la regresión con efectos fijos en el ámbito nacional, las variables edad, años de escolaridad, estado conyugal soltero y nivel de ingreso de más de 5 salarios mínimos tienen una elasticidad negativa y significativa respecto a la población ocupada manufacturera. Así, estas características tienen una relación negativa con la ocupación en las manufacturas. Estas mismas variables, excepto el nivel de ingreso de más de cinco salarios mínimos, mantienen elasticidades negativas y significativas en el modelo de efectos fijos y dummies temporales.

Las variables con elasticidades positivas sobre la población ocupada manufacturera en ambos modelos con efectos fijos son el empleo formal, el nivel de ingreso de dos y hasta tres salarios mínimos, laborar en pequeños establecimientos, así como en medianos establecimientos. Un aspecto a destacar es que residir en el ámbito urbano (localidades de más de 2,500 habitantes) no resultó significativo en ninguno de los modelos del ámbito nacional. Estos resultados son congruentes con los encontrados en Pérez Santillán (2019b) mediante modelos logísticos multinomiales.

Con la misma metodología descrita se crearon cohortes, pero sólo se consideró la población en el Estado de México. La regresión para datos agrupados sólo registró la variable edad con elasticidad negativa significativa respecto a la población ocupada manufacturera. En este modelo, las variables años de escolaridad, empleo formal, niveles de ingreso de uno y hasta dos salarios mínimos; de dos y hasta tres salarios mínimos, población ocupada en unidades económicas con establecimiento y en establecimientos medianos mostraron elasticidades positivas y significativas sobre la población ocupada manufacturera. 


\section{CUADRO 3}

MÉXICO: POBLACIÓN OCUPADA MANUFACTURERA Y RELACIÓN CON CARACTERÍSTICAS DE LA POBLACIÓN

\begin{tabular}{|c|c|c|c|c|c|c|}
\hline & \multicolumn{3}{|c|}{$\begin{array}{l}\text { Nacional: Variable dependiente } \\
\text { Ocupado manufacturero }\end{array}$} & \multicolumn{3}{|c|}{$\begin{array}{l}\text { Estado de México: Variable } \\
\text { dependiente } \\
\text { Ocupado manufacturero }\end{array}$} \\
\hline & Pool & E. Fijos & E. Fijos + d & Pool & E. Fijos & E. Fijos + d \\
\hline \multirow[t]{2}{*}{ Sexo } & $-0.658^{* * *}$ & -0.243 & -0.202 & -0.0784 & 0.105 & 0.110 \\
\hline & $(0.232)$ & (0.289) & $(0.283)$ & (0.190) & $(0.261)$ & (0.259) \\
\hline \multirow[t]{2}{*}{ Edad } & $-0.974 * * *$ & $-0.700 * * *$ & $-0.713^{* * *}$ & $-0.333^{* * *}$ & $-0.564^{* * *}$ & $-0.573^{* * *}$ \\
\hline & $(0.0907)$ & $(0.146)$ & $(0.145)$ & $(0.0960)$ & $(0.122)$ & $(0.122)$ \\
\hline \multirow[t]{2}{*}{ Años de escolaridad } & $-0.232 * *$ & $-1.004^{* *}$ & $-0.999 * *$ & $0.231^{*}$ & -0.0560 & -0.0528 \\
\hline & $(0.103)$ & $(0.358)$ & $(0.354)$ & $(0.136)$ & $(0.315)$ & $(0.304)$ \\
\hline \multirow[t]{2}{*}{ Empleo formal } & $0.590 * * *$ & $0.576 * * *$ & $0.578 * * *$ & $0.365 * * *$ & $0.391^{* * *}$ & $0.398 * * *$ \\
\hline & $(0.0997)$ & $(0.123)$ & $(0.122)$ & $(0.0667)$ & $(0.0671)$ & $(0.0665)$ \\
\hline \multirow[t]{2}{*}{ Soltero } & $-0.311 * * *$ & $-0.178^{* *}$ & $-0.178 * *$ & -0.0324 & 0.00902 & 0.0115 \\
\hline & $(0.0543)$ & $(0.0768)$ & $(0.0773)$ & $(0.0441)$ & $(0.0433)$ & $(0.0417)$ \\
\hline \multirow[t]{2}{*}{ Hasta un SM } & 0.102 & 0.0778 & 0.0779 & 0.0431 & $0.0624^{*}$ & 0.0556 \\
\hline & $(0.0640)$ & $(0.0731)$ & $(0.0732)$ & $(0.0329)$ & $(0.0345)$ & $(0.0361)$ \\
\hline \multirow[t]{2}{*}{ De 1 hasta 2 SM } & -0.000213 & -0.0126 & -0.00194 & $0.131^{*}$ & $0.127 *$ & $0.128^{*}$ \\
\hline & $(0.0872)$ & $(0.115)$ & $(0.122)$ & $(0.0677)$ & $(0.0678)$ & $(0.0686)$ \\
\hline \multirow[t]{2}{*}{ De 2 hasta 3 SM } & $0.199 * * *$ & $0.133^{* *}$ & $0.123^{*}$ & $0.133^{* *}$ & $0.106^{* *}$ & $0.100 * *$ \\
\hline & $(0.0489)$ & $(0.0548)$ & $(0.0580)$ & $(0.0552)$ & $(0.0423)$ & $(0.0434)$ \\
\hline \multirow[t]{2}{*}{ Más de 5 SM } & $-0.145^{* * *}$ & $-0.136^{*}$ & -0.130 & 0.0139 & -0.0130 & -0.0132 \\
\hline & $(0.0479)$ & $(0.0737)$ & $(0.0768)$ & $(0.0183)$ & $(0.0138)$ & $(0.0131)$ \\
\hline \multirow[t]{2}{*}{ Con establecimiento } & -0.0408 & -0.0688 & -0.0791 & $0.315^{* * *}$ & $0.241 * *$ & $0.238^{* *}$ \\
\hline & $(0.0912)$ & $(0.0987)$ & (0.0979) & $(0.0641)$ & $(0.0843)$ & $(0.0842)$ \\
\hline \multirow[t]{2}{*}{ Pequeños establecimientos } & $0.0893 * *$ & $0.129 * *$ & $0.128 * *$ & 0.00255 & 0.0234 & 0.0246 \\
\hline & $(0.0364)$ & $(0.0575)$ & $(0.0587)$ & $(0.0333)$ & $(0.0460)$ & $(0.0461)$ \\
\hline \multirow[t]{2}{*}{ Medianos establecimientos } & 0.0659 & $0.0894 * *$ & $0.0854 * *$ & $0.172 * * *$ & $0.126 * * *$ & $0.127^{* * *}$ \\
\hline & $(0.0530)$ & $(0.0350)$ & $(0.0357)$ & $(0.0447)$ & $(0.0324)$ & $(0.0333)$ \\
\hline \multirow[t]{2}{*}{ Ámbito urbano } & -0.0740 & -0.216 & -0.143 & 0.0805 & -0.245 & -0.233 \\
\hline & $(0.265)$ & $(0.600)$ & $(0.628)$ & $(0.435)$ & $(0.576)$ & $(0.547)$ \\
\hline \multirow[t]{2}{*}{ Constant } & $1.979 * * *$ & $3.019 * * *$ & $3.089 * * *$ & 0.623 & $2.015^{* * *}$ & $2.032 * * *$ \\
\hline & $(0.348)$ & $(0.915)$ & $(0.936)$ & $(0.570)$ & $(0.650)$ & $(0.640)$ \\
\hline Observaciones & 887 & 887 & 887 & 858 & 858 & 858 \\
\hline R-squared & 0.967 & 0.825 & 0.826 & 0.819 & 0.442 & 0.444 \\
\hline Número de cohortes & & 17 & 17 & & 17 & 17 \\
\hline Años de escolaridad promedio & 8.4 & & & & & 9.9 \\
\hline
\end{tabular}

Errores estándar robustos entre paréntesis

*** $p<0.01, * * p<0.05,{ }^{*} p<0.1$

Fuente: estimaciones propias. 
En el modelo de efectos fijos para la población ocupada manufacturera en el Estado de México, nuevamente la edad resultó con elasticidad negativa y significativa. Las variables empleo formal, los niveles de ingreso de hasta un salario mínimo, de uno y hasta dos salarios mínimos, y de dos y hasta tres salarios mínimos, así como laborar en unidades económicas con establecimiento y con establecimientos medianos arrojaron elasticidades positivas y significativas. En el modelo con efectos fijos y temporales para la población ocupada manufacturera en el Estado de México sobresale que se mantiene la edad como la única variable con elasticidad negativa, mientras se conservan las variables con elasticidad positiva y significativas, con excepción del nivel de ingreso de hasta un salario mínimo. De nuevo, es útil observar los resultados en otros ejercicios empíricos para la población ocupada manufacturera en el Estado de México.

En Pérez Santillán (2019b), mediante regresiones logísticas multinomiales se encontró que a mayor edad, disminuye la probabilidad de ser ocupado manufacturero para 2005 y 2018, resultado en el mismo sentido que lo encontrado con el método de pseudopanel. Igualmente, ser del sexo masculino incrementa la probabilidad de ser ocupado manufacturero en 2005 y la disminuye en 2018. En tanto, mayor escolaridad disminuye la probabilidad de ser ocupado manufacturero, 0.006 y 0.011 de acuerdo con los modelos para 2005 y 2018 , respectivamente. Además, para 2005 y 2018 las personas con ingresos mayores a un salario mínimo y hasta dos salarios mínimos presentaron una mayor probabilidad de ser ocupado manufacturero, comparados con aquellas personas con ingresos de hasta un salario mínimo; mientras niveles de ingreso de dos salarios mínimos o más reducen la probabilidad de ser ocupado manufacturero. Esto es congruente con lo encontrado en el pseudo panel, en el cual se encontraron elasticidades positivas para los niveles de ingreso de uno y hasta dos salarios mínimos en relación con la población ocupada manufacturera. Finalmente, para 2005 y 2018, un mayor tamaño de establecimiento incrementa la probabilidad de ser ocupado manufacturero, lo que es congruente con lo encontrado mediante el uso de pseudo panel. 


\section{CONCLUSIONES}

Entre el primer trimestre de 2005 y el tercer trimestre de 2019, el Estado de México se mantiene como la principal entidad por su participación en la población ocupada manufacturera, con $15.2 \%$ del total nacional. Durante el periodo, la entidad también se caracterizó por ser una de las que mayores incrementos de población ocupada manufacturera registró, solo detrás de Guanajuato.

El empleo manufacturero ha crecido por debajo del crecimiento del empleo total, por lo que a nivel nacional y para el Estado de México ha perdido participación en la estructura del empleo. En el Estado de México, en el periodo de estudio, las personas ocupadas en manufacturas pasaron de 1.13 a 1.36 millones, equivalente a un incremento de $21.0 \%$ (237 mil personas), porcentaje menor al incremento nacional. En términos relativos el crecimiento del empleo manufacturero, fue menor al observado para el total del empleo (37.5\%), y ese crecimiento representó sólo $11.5 \%$ del incremento total en el empleo, por lo que la participación del empleo manufacturero en la entidad pasó de $20.5 \%$ a $18.1 \%$ del empleo total, y pese al descenso aún está por encima de la participación a nivel nacional.

Son escasas las investigaciones que han abordado el tema de las características de la población en edad de trabajar, que se relacionan con la evolución de la población ocupada manufacturera. Para avanzar en este tema, en este trabajo se propuso aproximar la relación entre algunas características de la población y los ocupados en manufacturas. Así como la descripción de indicadores del mercado laboral nacional y en el Estado de México muestra peculiaridades que comparten, también destacan algunas diferencias y similitudes en los resultados del modelo propuesto.

Con base en datos de la ENOE, se construyó un pseudopanel. Los resultados de la regresión con efectos fijos en el ámbito nacional muestran que, las variables edad, años de escolaridad, estado conyugal soltero y nivel de ingreso de más de 5 salarios mínimos tienen una elasticidad negativa y significativa respecto a la población ocupada manufacturera. Este conjunto de variables presenta una relación negativa con las personas ocupadas en manufacturas. Estas mismas variables, excepto el nivel de ingreso de más de cinco salarios mínimos, mantienen elastici- 
dades negativas y significativas en el modelo de efectos fijos y dummies temporales.

Las variables con elasticidades positivas sobre la población ocupada manufacturera en ambos modelos con efectos fijos son el empleo formal, el nivel de ingreso de dos y hasta tres salarios mínimos, laborar en pequeños establecimientos, así como en medianos establecimientos.

La metodología de pseudopanel se aplica a datos del Estado de México. En este caso, en el modelo de efectos fijos, la edad nuevamente resultó con elasticidad negativa y significativa. Las variables empleo formal, niveles de ingreso de hasta un salario mínimo, de uno y hasta dos salarios mínimos, y de dos y hasta tres salarios mínimos, así como laborar en unidades económicas con establecimiento y con establecimientos medianos arrojaron elasticidades positivas y significativas. En el modelo con efectos fijos y temporales para la población ocupada manufacturera en el Estado de México sobresale que se mantiene la edad como la única variable con elasticidad negativa, mientras se sostienen las variables con elasticidad positiva y significativas con excepción del nivel de ingreso de hasta un salario mínimo.

Lo resultados anteriores constituyen un primer acercamiento necesario para un panorama de variables que se asocian a la evolución de la ocupación en manufacturas. Los resultados sugieren que se debe revisar el tema, porque a mayor edad, es menor la participación en la ocupación manufacturera. Este hecho podría estar limitando la entrada a ocupaciones manufactureras a personas de mayor edad. Adicionalmente, se requiere poner atención al tipo de ocupaciones generadas en manufacturas, pues a mayor logro educativo (medido por los años promedio de escolaridad), la relación es negativa con la ocupación manufacturera, lo que indicaría que los empleos manufactureros tienden a ser de requerimientos de menor cualificación. Este resultado ya se ha presentado en otros trabajos, como Huesca y Padilla (2012) y Calderón et al. (2017); estos últimos señalan que existe un círculo vicioso inducido por el tipo de tecnología que impera en las manufacturas y que tiende a simplificar los procesos de trabajo y a favorecer la demanda de trabajo poco calificado en detrimento del calificado. Destaca el descenso en la demanda de habilidades, por lo que en el sector manufacturero el trabajo calificado no encuentra empleos acordes con su formación, a pesar de mantenerse una alta tasa de rentabilidad media de la escolaridad. 
Por otro lado, si bien las actividades manufactureras no son las que generan menores ingresos, el análisis indica que los niveles de ingreso de hasta dos salarios mínimos se asocian positivamente con la ocupación manufacturera. Al respecto, Escobar (2011) destacaba la relación inversa entre salarios y crecimiento del empleo manufacturero, mientras De Jesús et al (2018), para un ejercicio con un periodo más cercano al de este trabajo, señalaban que no existía evidencia de que el incremento en los salarios condujeran al descenso en la generación de empleos. No obstante, los resultados del presente trabajo se encuentran en línea con la precarización de la ocupación en términos salariales observados en años recientes, o de otra forma, el incremento de empleo con bajos niveles de ingreso. Aunque se debe considerar que los incrementos recientes en el salario mínimo han incidido en mejorar los niveles de ingreso asociados a cada agrupación en términos de salarios mínimos. Además de las medidas que han favorecido el incremento del salario mínimo, se requiere revisar el tipo de actividades que lleva a la generación de empleos con esa característica en los ingresos laborales, de manera que se propongan medidas de política que promuevan empleos con mejores remuneraciones.

Aún está pendiente profundizar en las relaciones encontradas, así como en su variación entre las entidades federativas. Avanzar en el conocimiento de las características de la población que se asocian positiva o negativamente con la ocupación manufacturera permitirá generar insumos para proponer medidas tendientes a enriquecer las condiciones del empleo manufacturero. Para mejorar aún más esta situación, se requiere ahondar en el análisis y generar políticas públicas específicas con base en la evidencia encontrada. 


\section{BIBLIOGRAFÍA}

Calderón Villarreal, C, G. L. Ochoa-Adame y L. Huesca-Reynoso (2017). "Mercado laboral y cambio tecnológico en el sector manufacturero mexicano (2005-2014)", Economía, sociedad y territorio, vol. 17, núm. 54, pp. 523-560.

Carbajal Suárez, Y. y L. de Jesús Almonte (2017). “Empleo manufacturero en la Región Centro de México. Una estimación por gran división”, Contaduría y Administración. vol. 62, núm. 3, pp. 880-901.

Carbajal Suárez, Y. y B. Carrillo Macario (2017). "Producción y empleo manufacturero en las entidades federativas de México 1988-2013”, en Y. Carbajal Suárez y L. de Jesús Almonte (coords.). Dinámica del empleo y la producción manufacturera en México. Ediciones Eón-Universidad Autónoma del Estado de México. México, pp. 15-41.

Carbajal Suárez, Y. y B. Carrillo Macario (2017). "El empleo en los subsectores de la manufactura en las entidades federativas de la Región Centro de México 1998-2014”. Paradigma económico, vol. 8, núm. 1. pp. 77-105.

Coneval (Consejo Nacional de Evaluación de Política de Desarrollo Social) (2016). "Coneval presenta información referente al índice de la tendencia laboral de la pobreza al tercer trimestre de 2019". <https://www.coneval. org.mx/Medicion/Paginas/ITLP-IS_resultados_a_nivel_nacional.aspx> (12 de enero 2020).

De Jesús Almonte, L., M. E. Morales Fajardo y Y. Carbajal Suárez (2018). “Inversión extranjera directa y empleo manufacturero. Un análisis regional con datos de panel para México, 2007-2014". Papeles de Población. vol. 24, núm. 96. pp. 187-216.

Deaton. A. (1985). "Panel data from a time series of cross-sections", Journal of Econometrics vol. 30, núm. 1-2, pp. 109-126.

Dussel Peters, E. y L. Pérez Santillán (2018). La cadena del calzado y la generación de empleo: cantidad y calidad (2000-2018). México, CICEG / CECHIMEX / UNAM.

Escobar Mendez, A. (2011). "Determinantes del empleo en la industria manufacturera en México", Papeles de Población. vol. 17, núm. 67. pp. 251276.

INEGI (Instituto Nacional de Estadística y Geografía) 2007. “Cómo se hace la ENOE. Métodos y procedimientos”. <https://www.snieg.mx/ DocumentacionPortal/iin/Acuerdo_6_V/ENOE_como_se_hace_la_ ENOE1\%5B1\%5D.pdf> (diciembre de 2019).

Llamas Huitrón, I. y N. Garro Bordonaro (2003). "Trabajo, formalidad, escolaridad y educación”, en E. De la Garza y C. Salas (coords.). La situación del trabajo en México, 2003. UAM-IET/Solidarity Center/Plaza y Valdés. México, pp.1-28. 
Martínez Luis, D., I. Caamal Cauich, J.A. Ávila Dorantes y L. A. Pat Fernández (2018). "Política fiscal, mercado de trabajo y empleo informal en México", Revista Mexicana de Economía y Finanzas. vol.13, núm. 1, pp. 77-98. México.

Mckenzie, D. (2004). “Asymptotic Theory for Heterogeneous Dynamic Pseudo-Panels". Journal of Econometrics, núm. 2, vol. 120, pp. 235-262.

McConnell, C., S. Brue y D. Macpherson (2003). Economía Laboral, sexta edición, editorial McGraw Hill.

Okun, A. (1962). "Potential GNP: Its Measurement and Significance", Economics for Policymaking. MIT Press, Cambridge, MA.

Perera, M., C. González y J. Ramírez (2011). "El mercado laboral paraguayo: un análisis del comportamiento del empleo y los ingresos entre 19972008.", en Borda, Dionisio (Coord.) Comportamiento del empleo e ingresos en el Paraguay. análisis de una década (1997-2008). Centro de Análisis y Difusión de la Economía Paraguaya, Paraguay, pp 112-157.

Pérez Santillán, L. (2019a). "Calidad y cantidad de empleo: Principales características a nivel nacional y en el Estado de México". 7 ${ }^{\mathrm{a}}$. Jornada Multidisciplinaria Unidad Académica Profesional Huehuetoca, UAEMex, 23 abril. $<$ https://www.academia.edu/39253052/Calidad_y_cantidad_LPS $>$.

Pérez Santillán, L. (2019b). "El empleo en las manufacturas: características a nivel nacional y en el Estado de México", presentación de avance de investigación. México, Facultad de Economía, UAEMEX, octubre. $<$ https://www.academia.edu/40714974/Empleo_en_las_manufacturas caracter\%C3\%ADsticas $>$ (diciembre 2019).

Huesca Reynoso, L. y M. Padilla Arriola (2012). "Empleo, escolaridad y sector informal en la frontera norte de México y Chihuahua: expectativas de ocupación en la crisis", Ensayos Revista de Economía, Vol.31. Núm. 2. pp. 57-86.

Ríos Almodóvar, J. G. y S. Carrillo Regalado (2014). "El empleo calificado y no calificado en la manufactura de México ante la crisis de 2009", Economía, Sociedad y Territorio, vol. 14, núm. 46, pp. 687-714.

Sánchez, A., V. Villarespe Reyes, D. A. Román Cedillo y A. L. Herrera Merino (2016). "Determinantes de las horas de trabajo de las mujeres en México: un enfoque de pseudopanel (2005-2010)", Revista CEPAL, núm. 120, diciembre, pp. 127-139.

Varela Llamas, R. y M. Y. Nava Rubio (2015). "Determinantes de la búsqueda de empleo desde la ocupación: una estimación Logit Multinomial”, Estudios sociales. 2015, vol. 23, núm. 45. pp. 83-111.

Vásquez Núñez, J. (2018). "Crecimiento económico, estructura del mercado laboral, pobreza y desigualdad por ramas de actividad económica". Documento de trabajo, núm. 243. Departamento de Política de Empleo. Ginebra, OIT. 